\title{
Peróxido de Hidrógeno al $35 \%$ y su efecto sobre la microdureza dental
}

\section{5\% Hydrogen Peroxide and its effect on dental microhardness}

TORRES-CAPETILLO, Evelyn Guadalupe $\dagger$, CAPETILLO-HERNANDEZ, Guadalupe Rosalía*, LECOURTOIS-AMÉZQUITA, Mariana Gabriela y TIBURCIO-MORTEO, Leticia

\author{
Universidad Veracruzana, Facultad de Odontología, Región Veracruz
}

ID $1^{\mathrm{er}}$ Autor: Evelyn Guadalupe, Torres-Capetillo / ORC ID: 0000-0003-0576-0327, Researcher ID Thomson: T-16802018, CVU CONACYT ID: 308188

ID $1^{\text {er }}$ Coautor: Guadalupe Rosalía, Capetillo-Hernández, ORC ID: 0000-0002-2033-4660, Researcher ID Thomson: S7875-2018, CVU CONACYT ID: 386320

ID $2^{\text {do }}$ Coautor: Mariana Gabriela Lecourtois Amézquita / ORC ID: 0000-0002-4633-6023, CVU CONACYT ID: 297188

ID $3^{\text {er }}$ Coautor: Leticia Tiburcio Morteo / ORC ID: 0000-0003-1806-4355

DOI: $10.35429 / J O H S .2020 .23 .7 .1 .4$

Recibido Agosto 10, 2020; Aceptado Diciembre 03, 2020

\section{Resumen}

Los peróxidos son actualmente los productos más utilizados para el aclaramiento dental, cuando el peróxido se descompone produce una disminución del $\mathrm{pH}$ en el medio en el que se encuentra, de forma inmediata en el esmalte produce grabado ácido que varía en función del pH del producto, tiempo de contacto y concentración. Objetivos: Conocer el efecto del peróxido de hidrógeno al $35 \%$ sobre la microdureza dental. Metodología: Estudio experimental, transversal, descriptivo. Empleando 50 dientes que fueron cortados $1 \mathrm{~mm}$ por debajo de la unión amelocementaria para crear dos caras vestibular y palatina. Se le sometió a un aclaramiento dental a base de peróxido de hidrógeno al $35 \%$, para saber cuánta microdureza pierde el esmalte, unidad de medida Vickers. La micro dureza se midió después de realizar 3 indentaciones en diferente superficie del esmalte a una fuerza de 50 gramos y a un tiempo de 20 segundos con una punta de diamante que dejaba una huella observada en el microscopio en forma de rombo piramidal; basándose en las diagonales vertical y horizontal se determinaba la microdureza Vickers. Contribución: De acuerdo con los resultados obtenidos encontramos que la aplicación de peróxido de hidrógeno al 35\% disminuyó en un $25.08 \%$ la microdureza dental.

Peróxido de hidrógeno, Microdureza, Esmalte

\begin{abstract}
Peroxides are currently the most widely used products for tooth whitening, when peroxide decomposes it produces a decrease in the $\mathrm{pH}$ in the medium in which it is found, immediately in the enamel produces acid etching that varies depending on the $\mathrm{pH}$ of the product, contact time and concentration. Objectives: To know the effect of hydrogen peroxide at $35 \%$ on dental microhardness. Methodology: Experimental, transversal, descriptive study. Using 50 teeth that were cut $1 \mathrm{~mm}$ below the amelocemental junction to create two vestibular and palatal faces. The teeth were lightened with $35 \%$ hydrogen peroxide to find out how much micro-hardness the enamel loses, the Vickers unit of measurement. The microhardness was measured after making 3 indentations in different surfaces of the enamel at a force of 50 grams and a time of 20 seconds with a diamond tip that left a trace observed under the microscope in the form of a pyramidal diamond; based on the vertical and horizontal diagonals, the Vickers microhardness was determined. Contribution: According to the results obtained, we found that the application of $35 \%$ hydrogen peroxide decreased dental microhardness by $25.08 \%$.
\end{abstract}

Hydrogen peroxide, Microhardness, Enamel

Citación: TORRES-CAPETILLO, Evelyn Guadalupe, CAPETILLO-HERNANDEZ, Guadalupe Rosalía, LECOURTOISAMÉZQUITA, Mariana Gabriela y TIBURCIO-MORTEO, Leticia. Peróxido de Hidrógeno al 35\% y su efecto sobre la microdureza dental. Revista de Ciencias de la Salud. 2020. 7-23: 1-4

\footnotetext{
* Correspondencia del Autor (gcapetillo@uv.mx)

$\dagger$ Investigador contribuyendo como primer Autor
} 


\section{Introducción}

El blanqueamiento dental es una de las técnicas odontológicas más utilizadas en la actualidad, produciendo en ocasiones resultados no predecibles, por variaciones en la respuesta de los agentes utilizados para tal efecto. Esta rama relacionada a la Odontología estética y relacionada con varias especialidades del área ha generado cada vez más conocimientos sobre los principios activos que llevan a un blanqueamiento exitoso, abriéndose nuevos campos investigativos que mejoren las expectativas de los profesionales y de los pacientes que solicitan dicho procedimiento. Mejía (2012).

El peróxido de hidrógeno actúa como un agente oxidante que tiende a captar los electrones del medio quedando reducido; mientras que las moléculas de pigmentos de cadenas moleculares largas que se encuentran inmersas en la estructura funcionan como un agente reductor que suministra electrones de su estructura química al medio siendo oxidado, generándose un rompimiento de enlaces simples y dobles de las cadenas conjugadas extensas. Baldión P. 2013.

El esmalte no es considerado un tejido, ya que no posee células dentro de la sustancia mineralizada, sino más bien es un casco que da protección al resto de los tejidos que conforma el diente, es llamado también sustancia adamantina.

El esmalte está formado por un $96 \%$ de sales minerales que forman cristales de hidroxiapatita, el cual esta densamente empaquetados siendo colocados uno de bajo del otro por aposición, dando origen a los prismas como unidades básicas, al finalizar su formación, los ameloblastos forman una capa selladora sin presencia de prismas sino solo cristales aprismáticos.

El proceso químico de blanqueamiento dental consiste en la reacción de óxidoreducción, a través de la cual la cantidad de pigmentos removidos es proporcional a la concentración y el tiempo de exposición del esmalte al agente blanqueador. Da D, Costa S, 2017.
Las moléculas con dobles enlaces conjugados absorben selectivamente algunas longitudes de onda de luz y no absorben otras longitudes de onda, lo que produce el efecto de un diente más oscuro. Al usar un peróxido para romper los dobles enlaces conjugados en enlaces simples y moléculas más pequeñas, estas moléculas se vuelven translúcidas y la discromía tiende a desaparecer. Zerón A, 2018.

Hoy en día existen diferentes agentes blanqueadores, como son: el peróxido de hidrógeno, el peróxido de carbamida y el perborato de sodio. Cada uno presenta concentraciones variadas, pero en definitiva es el peróxido de hidrógeno quien inicia el proceso de degradación de las moléculas orgánicas complejas y de elevado peso molecular, que reflejan una longitud de onda de la luz específica, causante del color de la mancha. A través de este mecanismo de oxidación se logran estructuras de carbono hidrófilas, no pigmentadas y con enlaces de carbono saturados. López N, 2016

El peróxido de carbamida se disocia en peróxido de urea y peróxido de hidrógeno. Por otra parte, el perborato de sodio se descompone, una vez en contacto con el agua en: agua, metaborato de sodio y peróxido de hidrógeno, siendo en ambos casos el peróxido de hidrógeno el agente activo. López N, 2016.

Es importante considerar que el blanqueamiento presenta algunas limitaciones, considerando que es una alternativa formidable en el tratamiento estético. Independientemente de la técnica a ser utilizada es necesaria la supervisión y acompañamiento de un Cirujano Dentista, pues con un criterio diagnóstico adecuado, el tratamiento y la seguridad del mismo estarán garantizados y los resultados satisfactorios serán alcanzados.

\section{Objetivo}

Conocer el efecto del peróxido de hidrógeno al $35 \%$ sobre la microdureza dental. 


\section{Metodología}

Estudio de tipo experimental, descriptivo, transversal, cuantitativo en la Facultad de Odontología y de Ingeniería a 50 especímenes (25 GC y 25 GE) al GE se les aplicó peróxido de hidrógeno al $35 \%$ y se midió con un microdurómetro su microdureza dental medida en Vickers. Se realizó el análisis estadístico descriptivo mediante el programa Excel (Microsoft, versión 7) y el programa SPSS.

El producto de blanqueamiento que se ocupó para realizar este estudio fue Pola Office al 35\% de peróxido de hidrógeno.

Para conocer la microdureza de cada diente se realizaron 3 indentaciones en diferente superficie del esmalte a una fuerza de 50 gramos y a un tiempo de 20 segundos con una punta de diamante que dejaba una huella observada en el microscopio en forma de rombo piramidal; basándose en las diagonales vertical y horizontal se determinaba la microdureza Vickers mediante la fórmula siguiente: $\mathrm{HV}: 1.8544 \mathrm{P} / \mathrm{d}^{2}$ donde $\mathrm{HV}$ es la microdureza Vickers, $\mathrm{P}$ es la carga de prueba usada y d es el promedio calculado de las diagonales medidas en $\mathrm{mm}^{2}$.

\section{Resultados}

A partir de los resultados obtenidos se calculó el promedio y su desviación estándar, posteriormente se realizó una prueba de diferencia de medias. El promedio del grupo control fue de 302.05 Vickers y el promedio del grupo experimental fue de 226.32 Vickers.

Con base en los promedios obtenidos del grupo control y grupo experimental se encontró una pérdida de microdureza Vickers de $25.08 \%$ después de aplicar el agente blanqueador Pola Office al 35\% de peróxido de Hidrógeno.

\begin{tabular}{|c|c|c|c|c|c|}
\hline Grupos & Mediana & $\begin{array}{l}\text { Valor } \\
\text { Mínimo }\end{array}$ & $\begin{array}{c}\text { Valor } \\
\text { Máximo }\end{array}$ & $\begin{array}{l}\text { Desv. } \\
\text { Típica }\end{array}$ & $\mathrm{p}$ \\
\hline $\begin{array}{l}\text { Dureza } \\
\text { antes }\end{array}$ & 335.90 & 184.33 & 386.26 & 73.21 & \multirow{2}{*}{0.000} \\
\hline $\begin{array}{l}\text { Dureza } \\
\text { después }\end{array}$ & 225.86 & 53.3 & 333.6 & $\begin{array}{r} \pm \\
62.37\end{array}$ & \\
\hline
\end{tabular}

Tabla 1 P $<0.05$ Prueba de Wilcoxon
Se realizó una prueba para evaluar la normalidad de los datos obtenidos con la prueba de Kolmogorov Smirnov, se obtuvo un valor de probalidad de 0.13 , por lo tanto, se reporta el valor de la mediana y los valores máximos mínimos como medidas de tendencia central y la desviación típica como medida de dispersión.

Al no comprobar la normalidad de los datos se aplicó una prueba estadística no paramétrica para dos muestras relacionadas, la prueba de Wilcoxon. El valor de probabilidad fue de 0.000 (punto de corte $\mathrm{p}<0.05$ ) por lo tanto se puede concluir que el agente blanqueador disminuye la dureza de los dientes de acuerdo con el experimento llevado a cabo.

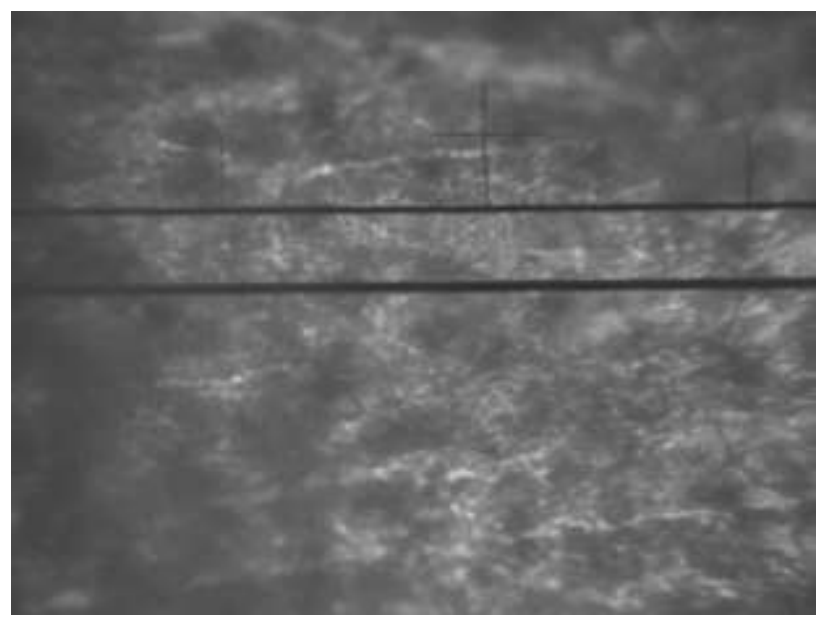

Figura 1 Figura 6.- Huella de indentación

\section{Discusión}

Ortíz y cols, 2016 demostraron que el blanqueamiento sí afecta la microbiología de la superficie del esmalte, produciendo porosidades, cráteres y depresiones, además de que disminuye su microdureza.

Chacko y cols, 2018 coincidió en que las irregularidades aumentan en la superficie del esmalte con sustancias químicas.

Vargas y cols, 2015 demostraron que las muestras blanqueadas con peróxido de carbamida al $38 \%$ presentaron la mayor pérdida de fosfato del esmalte dental, equivalente a una disminución de aproximadamente el $45 \%$ la cual ocurrió principalmente en las primeras dos semanas del estudio. 


\section{Conclusiones}

Se demostró que el blanqueamiento sí afecta la microbiología de la superficie del esmalte, produciendo porosidades, cráteres y depresiones, además de que disminuye su microdureza.

Las irregularidades de la superficie aumentaron con sustancias químicas. La presente investigación coincidió con las investigaciones Vargas y cols, 2015 donde las muestras blanqueadas con peróxido de carbamida al $38 \%$ presentaron la mayor pérdida de fosfato del esmalte dental, equivalente a una disminución de aproximadamente el $45 \%$ la cual ocurrió principalmente en las primeras dos semanas del estudio.

El presente estudio coincide con el de Domínguez y cols, 2013 que presentó una diferencia significativa $(\mathrm{p}<0.001)$ entre los valores de antes y después de ambos grupos, incrementando los valores de microdureza Vicker en el grupo GCC y disminuyendo en el $\mathrm{GC}$ en un $6 \%$ cada uno.

\section{Recomendaciones}

De acuerdo a esta investigación se recomienda que los especímenes a estudiar se obtengan después de un blanqueamiento dental con el uso de fuentes híbridas LED/LÁSER ya que este tipo de blanqueamiento está muy de moda y hay mucha controversia ya que muchos autores recomiendan no utilizarlas.

\section{Referencias}

Baldión Elorza PA, Arcos Hurtado LC, Mora Portilla MA. Efecto de los fluoruros en la composición química del esmalte dental posblanqueamiento. Effect of Fluorides over the Chemical Composition of Tooth Dental Enamel Post Bleaching. Univ Odontol. 2011;30(65):419.

Baldión P. Influence of post-bleachInG tIme on a composIte resIn bond strenGth to enamel. Rev Fac Odontol Univ Antioquia. 2013;25(1):92116.
Bersezio C, Martín J, Herrera A, Loguercio A, Fernández E. The effects of at-home whitening on patients' oral health, psychology, and aesthetic perception. BMC Oral Health. 2018;18(1):1-10.

Chacko Kalliath, Archana Mukunda, Meera Pynadath, Vidya Venugopal JP. Comparison between the effect of commercially available chemical teeth whitening paste and teeth whitening paste containing ingredients of herbal origin on human enamel. Inst Post Grad Teach Res Ayurveda, Jamnagar. 2018;39(2):113-7.

Da D, Costa S, Marson FC, Luiz A, Briso F, Pascotto $\mathrm{C}$, et al. ANÁLISE laboratorial da quantidade de peróxido de hidrogênio e o ph nos enxaguantes bucais e pastas dentais. Brazilian J Surg Clin Res - BJSCR. 2017;18:49-53.

López N, Vale L, Coca A. Recromias en dientes vitales con cambio de coloración. Rev odontológica Vidaurreta [Internet]. 2016;4(10):25-34. Available from: http://revzoilomarinello.sld.cu/index.php/zmv/a rticle/view/875.

Nam SH, Kwun HS, Cheon SH, Kim HY. Effects of whitening toothpaste on color change and mineral contents of dental hard tissues. Biomed Res. 2017;28(9):3832-6.

Ortíz Aguilar M, Zavala Alonso NV, Patiño Marín N, Martínez Castañón GA, Ramírez González JH. Efecto del blanqueamiento y el remineralizante sobre la microdureza y micromorfología del esmalte dental. Rev ADM. 2016;73(2):81-7.

Rodrigo M, Mejia G. (2012). Blanqueamiento Dental en dientes tratados endodónticamente. Rev Actual clínica., 1:11, 35-40.

Vargas-Koudriavtsev T, Durán-Sedó R, SáenzBonilla P, Bonilla-Mora V, Guevara-Bertsch M, Jiménez-Corrales RA, et al. Efecto de agentes de blanqueamiento dental sobre la concentración de fosfato en el esmalte dental por medio de espectroscopia Raman. Rev Odontológica Mex [Internet]. 2015;19(4):232-9. Available from: http://linkinghub.elsevier.com/retrieve/pii/S187 0199X15000427.

Zerón A. Dejando las cosas claras. Just to clarify. Rev ADM 2018;75(1):4-6. 